Original Research Paper

\title{
Three-Dimensional in Bonded Joints: A Short Review
}

\author{
${ }^{1}$ Filippo Berto, ${ }^{2}$ Relly Victoria V. Petrescu and ${ }^{2}$ Florian Ion T. Petrescu \\ ${ }^{I}$ Department of Engineering Design and Materials, NTNU, Trondheim, Norway \\ ${ }^{2}$ ARoTMM-IFToMM, Bucharest Polytechnic University, Bucharest, (CE) Romania
}

\author{
Article history \\ Received: 20-10-2016 \\ Revised: 28-12-2016 \\ Accepted: 30-12-2016 \\ Corresponding Author: \\ Filippo Berto \\ Department of Engineering \\ Design and Materials, NTNU, \\ Trondheim, Norway \\ Email: berto@gest.unipd.it
}

\begin{abstract}
Distributions of stress 3D in the vicinity of the overlapping corners of joints with a single lap were investigated with accuracy at present the man. A $(\mathrm{Fe})$, model accurately Finite Element 3D has been carried out in order to investigate the intensity of the stress of adjustments in the plan and outside of stress by the width of the plate. The influences of the thickness of the adhesive and the Poisson distribution have been taken into account. Numerical results showed the presence of modes engaged has been detected with precision a sudden rise in the way the out-of-plan is very close to the free surface side of the fitting.
\end{abstract}

Keywords: Finite Element Analysis, 3d Model, Lap Joint, Notch Stress Intensity Factors

\section{Introduction}

The adhesive joints were investigated in detail in the last decades and many investigations on this subject of research can be found easily in the past and the recent literature.

A review range of analytical approach for the bonded joint with glue is reported in (da Silva et al., 2009a; 2009b).

That review showing that most of the analytical models are two-dimensional.

As it is well known in the literature, bonded joints with adhesive are characterized by areas of high concentration stress in the vicinity of the corners of the overlap. For a bi-material stress takes place singularity also in the case in which any geometric discontinuity is present on the component (Bogy, 1970).

The assessment of the plan in the field of stress in the joints of by bonding of different materials have been well studied, among others, (Lazzarin et al., 2002).

The wording of the analytical allows an exact description of the field of two-Dimensional stress (2D) in the case of joints with elastic properties similar.

In the framework of this investigation, threeDimensional (3D distributions by stress in the vicinity of the overlapping corners of joints with a single lap investigated.

A (FE) model exactly the finished item 3D has been carried out with the purpose of studying the distribution of a mode in the region emphasized the interdiction.
The influences of the thickness of the adhesive and the Poisson distribution are well taken into account here.

\section{Finite Element Modelling}

A three-dimensional model of a connection with a single round is built by the use of ANSYS.

The dimensions and the properties of the materials of the join with a single round are given in Fig. 1. Due to the symmetry of the knuckle housing along the width, only half of the model has been shaped. Different thicknesses of the adhesive $\left(\mathrm{h}_{\mathrm{adh}}=0.1,0.25\right.$ and $\left.0.5 \mathrm{~mm}\right)$ and the Poisson distribution $\left(v_{\mathrm{adh}}=0.1,0.35\right.$ and 0.499$)$ they have been examined. Materials taken into account for the analysis are a structural steel tariff for the adherend $(\mathrm{E}=206 \mathrm{GPa}$ and $v=0.30)$ and by an epoxy resin for adhesive $\left(E_{a d h}=3500 \mathrm{MPa}\right.$ and $v_{\text {adh }}=0.1,0.35$ and 0.499). A declaration has been taken into account in the interfaces between the adhesive and adherents.

The model is of mesh elements of 4 nodes (SOLID185) and a net of refined has been used in order to be able to capture the distribution of the tensions in the immediate vicinity of the corners overlap, corners A and B in Fig. 1c.

The comparison between the components of stress at the two interfaces and in the middle of the adhesive have shown higher values by stress at the lower interface, i.e., at the corner of the A.

The results reported here are for that point, which has been proved to be the most critical. A small distance $(0.005 \mathrm{~mm}$ away from the single point) is used here to calculate the stress factors of intensity. 


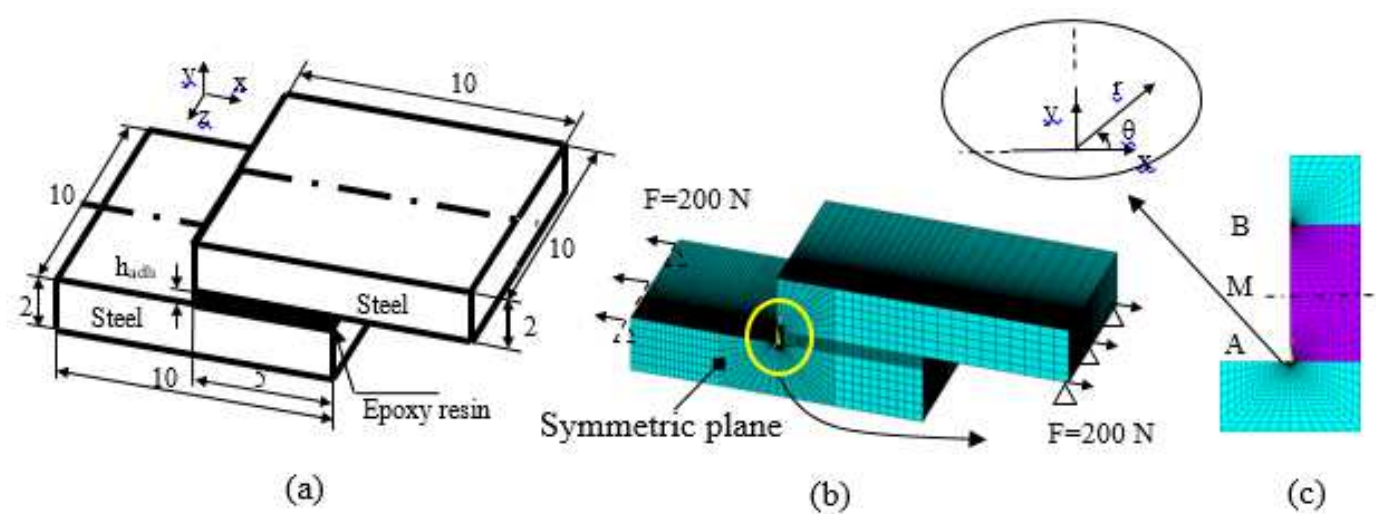

Fig. 1. Geometrical dimensions (in $\mathrm{mm}$ ) and materials of a single-lap joint (a); finite element model and boundary conditions (b); overlap corners and mid-plane path used for stress evaluation

\section{Results and Discussion}

\section{Stress Analysis in the Adhesive}

The confrontation with the sharp V nicks, the stress factors of intensity (notch NSIFs) are assessed along the line bisecting notch by hiring Gross and definitions Mendelsohn (Gross and Mandelson, 1972).

In the front of the apparent NSIFs are calculated in the area of the interface between the adhesive and slick crops.

The representatives of stress (singularity exponents) $\xi_{i}$ are different from those proposed by Williams for the materials mixing (Williams, 1952).

The main differences have been discussed in detail in the specialized literature, see Part No. (Lazzarin et al., 2002) and the references of the reported in this.

The variations in the stress as regards $\sigma_{\theta \theta}, \tau_{r \theta}$ and $\tau_{z \theta}$ and to the bottom of a joint faces with a single round, of a thickness of the adhesive $h_{a d h}=0.1 \mathrm{~mm}$ and the Poisson ratio $v_{a d h}=0.35$ are reported in Fig. 2 a.

The intensities of stress have been measured by the apparent-NSIFs, in the case where $K_{1}^{a p p}, K_{2}^{a p p}$ in Equation 1a and $1 \mathrm{~b}$ and $K_{3}{ }^{a p p}$ in the EC. (2), are designed here as simply an extension of the definition of gross and Mendelsohn.

Taking into account the mixing materials, a model analytically able to take into account the presence of the contemporary in the plan and out of the singularities of stress has been developed in (Lazzarin and Zappalorto, 2012).

This contribution has clarified, in theory, the reason for which the mode II and III, may not exists separately. The aim of this work is to study the influence of a mode, also for the geometry of the reported in Fig. 1.

The three to NSIFs are presented in Fig. $2 b$. Moreover, in order to document the better the variations in the A-NSIFs in the vicinity of the free surface side of the tour-knuckle housing, a distance of
$0.5 \mathrm{~mm}$ from the free surface is selected and the results are given in Fig. 2c:

$\left\{\begin{array}{l}K_{1}^{a p p}(z) \lim _{r \rightarrow 0} \sqrt{2 \pi} r^{\xi_{1}} \sigma_{\theta \theta} \\ K_{2}^{a p p}(z) \lim _{r \rightarrow 0} \sqrt{2 \pi} r^{\xi_{2}} \tau_{\theta \theta}\end{array}\right.$

(at the interface, $\theta=0$ in Fig. 1c)

$K_{3}^{a p p}(z) \lim _{r \rightarrow 0} \sqrt{2 \pi} r^{\xi_{3}} \tau_{z \theta}$

(at the interface, $\theta=0$ )

As shown in Fig. 2a all three components of stress $\sigma_{\theta \theta}$, $\tau_{r \theta}$ and $\tau_{z \theta}$ are holders, being the stress of zest the maximum.

The singularity indices of efforts to shear and peel are almost identical $\left(\xi_{1} \approx \xi_{2}=0.30\right)$.

The distributions of stress almost at the corner of the are plotted along the width of the joint Fig. 2a.

They prove that $\sigma_{\theta \theta}$ component of stress is dominant in a very wide range of the width of the common market.

However, in a very limited area near the surface side (approximately $0.05 \mathrm{~mm}$ from it), can be easily noticed an increase of how III component shear stress.

On the surface of the side, there may be effects similar edge singularitatile points of the corner (Berto et al., 2011; Pook, 1992; 2000).

A network $3 \mathrm{D}$ refined has been engaged in the area near the corner of overlapping; however, the requirement of balanced for $K_{3}{ }^{a p p}$, which should be zero at the free surface side, is only partially fulfilled. However, the mode $K_{3}{ }^{a p p}$ has been proved to be significantly reduced after reaching the maximum value.

\section{Effect of Adhesive Thickness}

The effect of the thickness of the adhesive (hadh) on how seemingly I and NSIFs mode III is presented in Fig. $3 a$. 


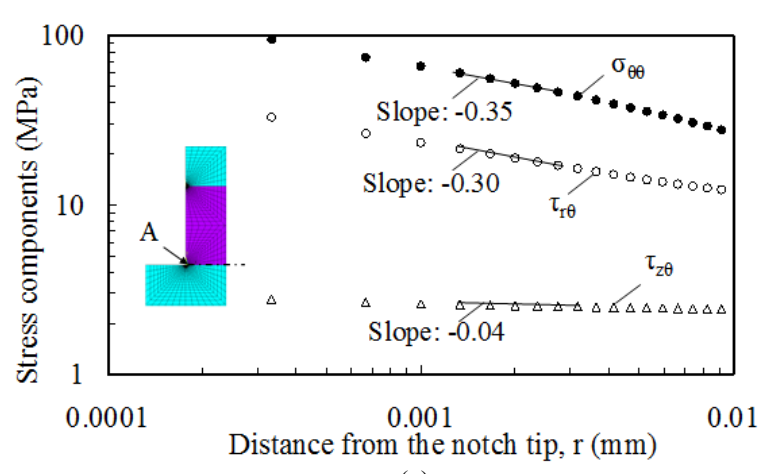

(a)

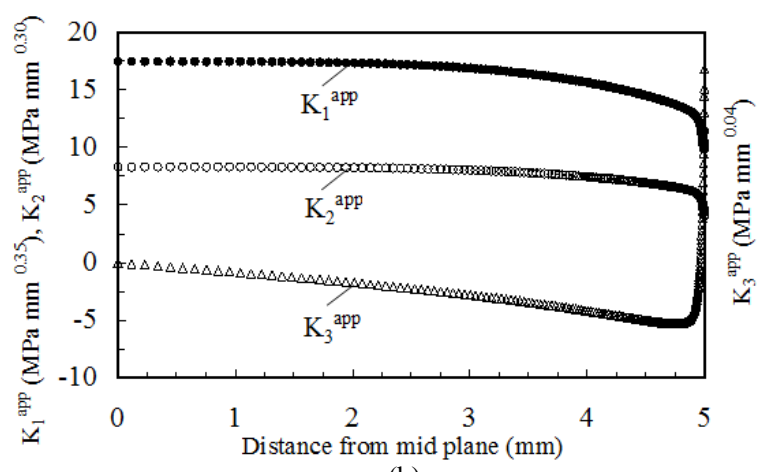

(b)

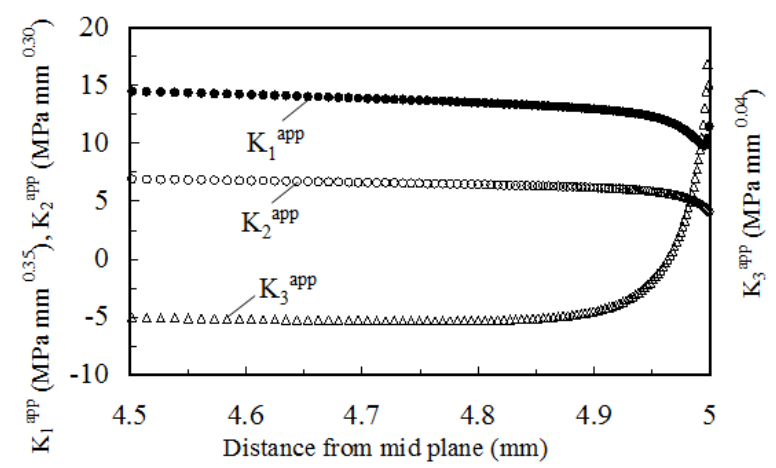

(c)

Fig. 2. Stress components along the adhesion line, as a function of the distance from point A, at the distance $z=0.5 \mathrm{~mm}$ from the lateral free surface of the joint (a); plots of the apparent NSIFs along the width of the joint (b) and in the vicinity of the lateral surface, $4.5 \leq z \leq 5.0 \mathrm{~mm}$, (c)

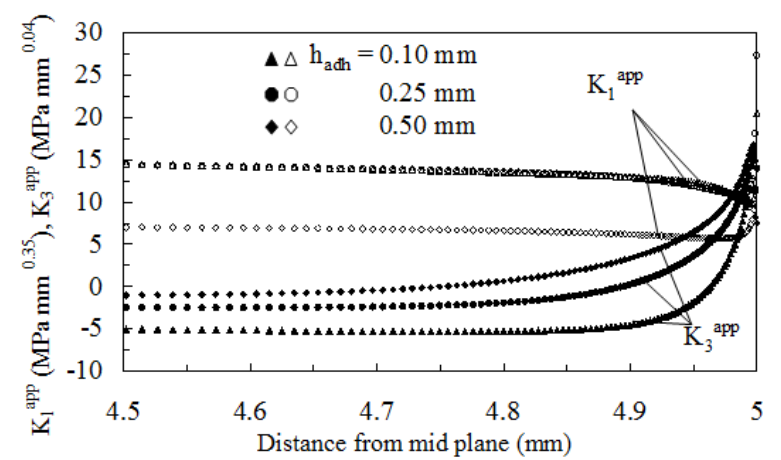

(a)

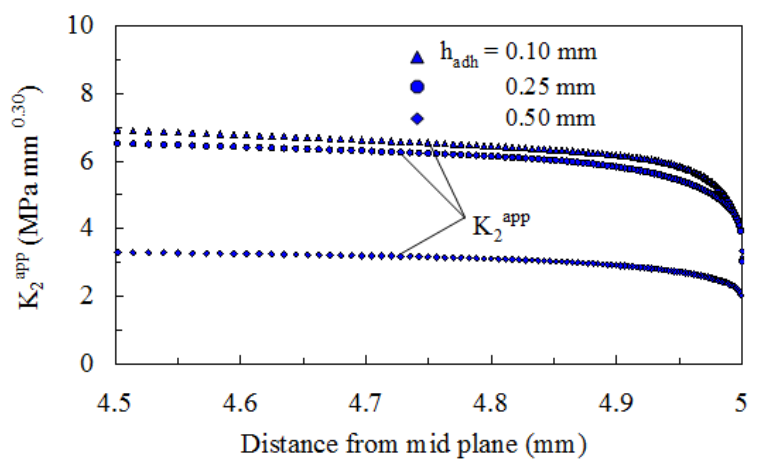

(b)

Fig. 3. Plots of the A-NSIFs along the width of the joint, at the lower interface, for different adhesive thicknesses; mode I and mode III (a); mode II (b)

The same effect is studied for mode II of Fig. 3b. In agreement with the Fig. $3 \mathrm{a}$ and $3 \mathrm{~b}$ and increase the thickness of the adhesive, $K_{1}{ }^{a p p}$ and $K_{2}{ }^{a p p}$ whereas increased $K_{3}{ }^{a p p}$, in particular, in the immediate vicinity of the surface.

More than that, a large variation of $K_{2}{ }^{a p p}$ is noted that a depending on the thickness of the adhesive, while limited is variation along the length of the largest part of the width of the bead.
However, the intensities of the stress in the plan to increase with increasing the thickness of the adhesive, it is for this reason joins with glue thicker are relatively weaker.

\section{Effect of adhesive Poisson's ratio}

The effect of the report the adhesive $\left(v_{a d h}\right)$ on the apparent NSIFs Poisson distribution is presented Fig. $4 \mathrm{a}$ and $4 \mathrm{~b}$ taking into account separately the modes IIII and II. 


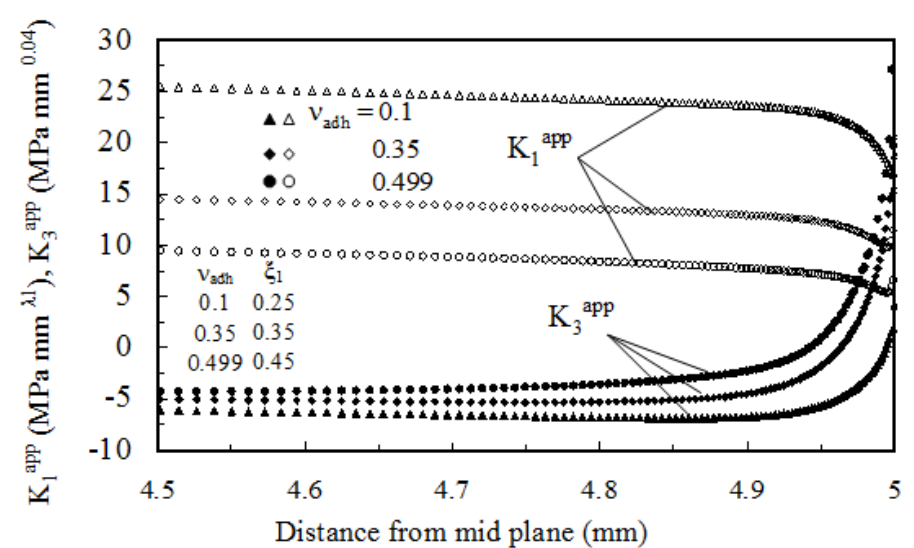

(a)

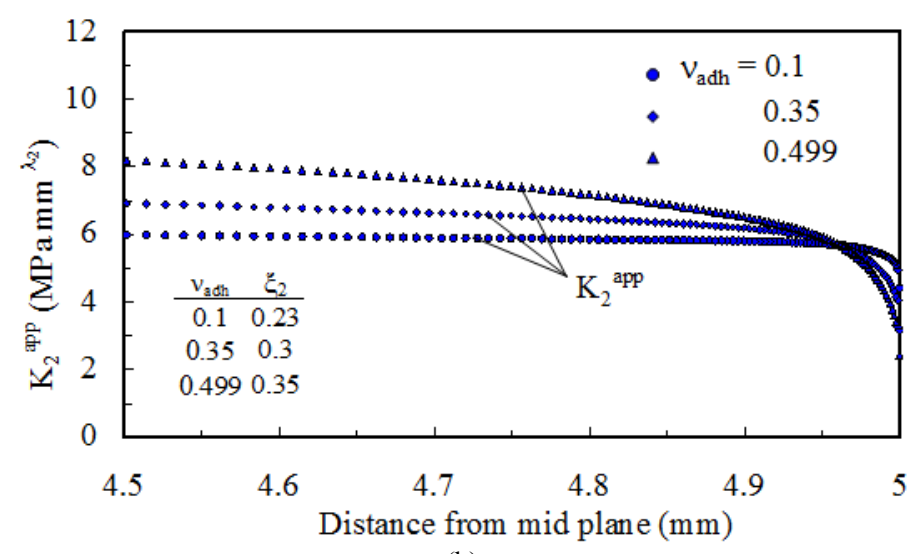

(b)

Fig. 4. Plots of the A-NSIFs along the width of the joint, at the lower interface, for different Poisson's ratio of the adhesive; mode I and mode III (a); mode II(b)

All parcels have a period of validity in the vicinity of the close to the exterior surfaces of the butt joint, $4.5 \mathrm{~mm}$ $\leq \mathrm{z} \leq 5.0 \mathrm{~mm}$.

In Fig. $4 \mathrm{a}$ and $4 \mathrm{~b}$ it is clear that the $\mathrm{K}_{2}{ }^{\text {app }}$ and $\mathrm{K}_{3}{ }^{\text {app }}$ increase as the ratio of the adhesive increases Poisson distribution (for $\mathrm{K}_{2}{ }^{\text {app }}$ this is valid for an area of very large width of adhesive, $0 \leq \mathrm{z} \leq 4.95 \mathrm{~mm}$ ). In the immediate vicinity of the surface of the side-free, the variation of the $\mathrm{K}_{2}{ }^{\text {app }}$ becomes strong and depends on the ratio between the adhesive of the Poisson distribution, Fig. $4 \mathrm{~b}$.

At the opposite pole is the trend of $\mathrm{K}_{1}{ }^{\mathrm{app}}$, which decreases as $v_{\text {adh }}$ decreases.

Variations of this parameter are once again in the main, the limited near the surface side free of joints by bonding $(\mathrm{z}>4.95 \mathrm{~mm})$.

Finally, it should be noted that the increase in the degree of singularitate varies in I and II, being of more than in the first case, in the case in which this varies $0.25-.45$ by changing $v_{\text {adh }}$ from 0.1 up to approximately 0.5 . Surprising, degree of singularitatii mode III proves to be almost constant $\left(\xi_{3} \approx 0.04\right)$, with the increase in the Poisson ratio adhesive.

\section{About Finite Element Method}

In numerical analysis, the Finite Element Method (EFM) is used to numerically solve partial differential equations.

For example, they can analytically represent the dynamic behavior of certain physical systems (mechanical, thermodynamic, acoustic, etc.).

Concretely, this allows to calculate numerically the behavior of even very complex objects, provided that they are continuous and described by a linear partial differential equation: Motion of a rope shaken by one of its ends, behavior a fluid arriving at high speed over an obstacle, deformation of a metal structure, etc.

In Fig. 5 one can see a two-dimensional solution of a magnetostatic equation obtained by finite elements (the lines give the direction of the field and the color its intensity).

In the Fig. 6 one can see the mesh used for the top image (the mesh is tightened around the area of interest). 


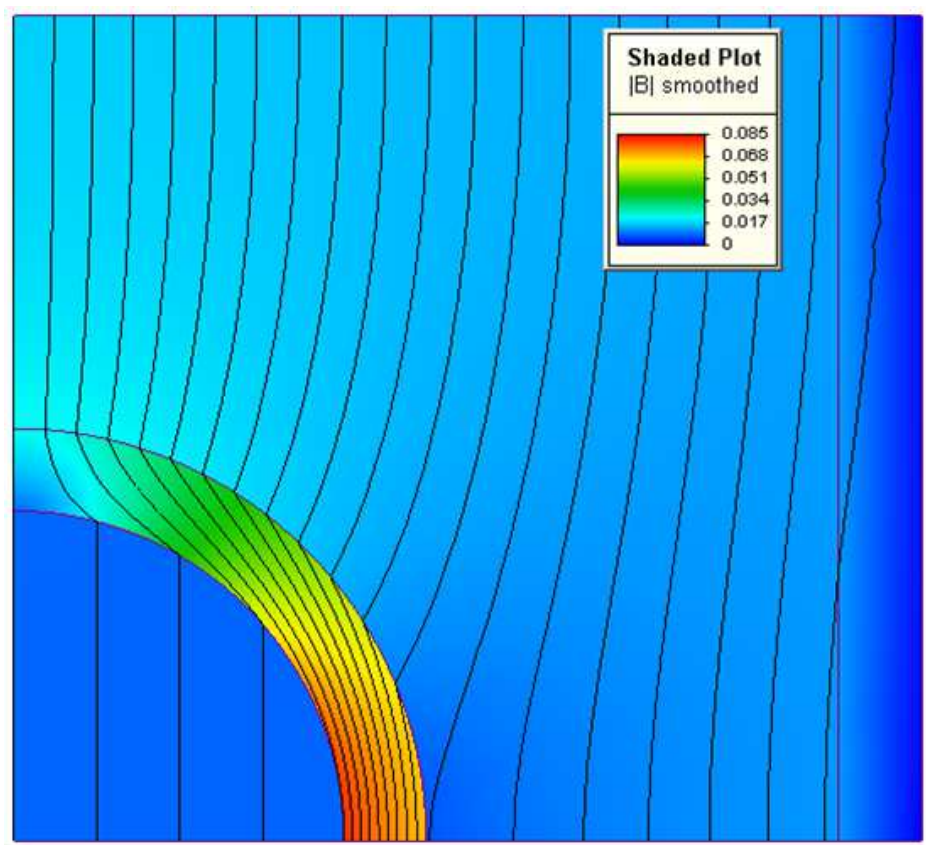

Fig. 5. Two-dimensional solution of a magnetostatic equation obtained by finite elements (the lines give the direction of the field and the color its intensity)

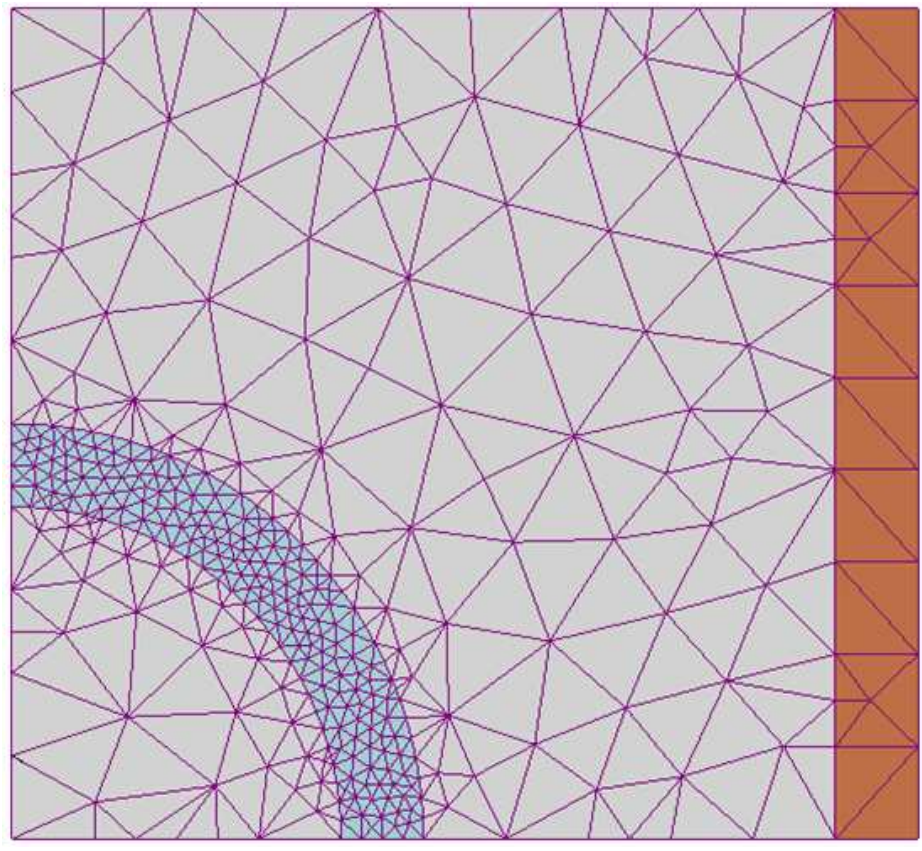

Fig. 6. Mesh used for the top image (the mesh is tightened around the area of interest)

The finite element method is part of applied mathematics. It is a question of implementing, using the principles inherited from the variational formulation or weak formulation, a mathematical discrete algorithm for finding an approximate solution of a Partial Differential Equation (or PDE) on a compact domain with Conditions at the edges and/or inside the compact.
Commonly referred to as Dirichlet (edge values) or Neumann (edge gradients) or Robin (gradient/edge values) conditions.

It is therefore above all the approximate resolution of a problem, where, thanks to the variational formulation, the solutions of the problem satisfy conditions of existence that are weaker than those of the solutions of 
the initial problem and where a discretization allows find an approximate solution. Like many other numerical methods, besides the algorithm of resolution in itself, arises the questions of quality of the discretization: Existence of solutions; Uniqueness of the solution; Stability; Convergence.

And of course: Measurement of error between a discrete solution and a unique solution of the initial problem. General framework of the finite element method as well as the most common practical case considering linear partial differential equations for which approximation by affine functions is sought.

One uses a part essentially physical and in particular mechanical. It should be considered only as a presentation of the constitutive elements of the discrete modeling used in resistance of materials via the finite element method. It is a very valid approach, a good pedagogical example. It brings a certain bias towards a more general approach, in particular because of the supposed linearity of the materials.

Consider a domain $\Omega$ (typically a portion of space) whose boundary is denoted by $\delta \Omega$ or $\Sigma$. We seek to determine a function $\mathrm{u}$ defined on $\Omega$, which is a solution of a Partial Differential Equation (PDE) for given boundary conditions. The PED describes the physical behavior of the system, for example, the laws of elasticity for a material resistance problem or Maxwell's equations for electromagnetism problems. The boundary conditions are constraints on the system. For example, for a material resistance problem, certain parts of the system are displaced, for example, it is required that a bearing zone is immobile and stresses are imposed on other zones (weight, pressure of contact...).

The method of the finite elements (MET) allows to solve in a discrete and approximate way this problem; an approximate "sufficiently" reliable solution is sought.

The discretization consists in "cutting" the domain $\Omega$, that is to say to seek a solution of the problem on a polygonal or polyhedral domain in pieces; there is therefore a redefinition of geometry. Once the geometry is approximated, it is necessary to choose an approximation space of the solution of the problem, in the MEF, this space is defined using the mesh of the domain (which also explains why it is necessary to approach the geometry). The mesh of the domain makes it possible to define a pavement of which the blocks are the finite elements.

On each of the finite elements, it is possible to linearize the EDP, that is to say to replace the equation with the partial derivatives by a system of linear equations, by approximation. This system of linear equations can be described by a matrix; there is therefore a matrix per finite element. However, boundary conditions are defined on the boundaries of the global system and not on the boundaries of each finite element; It is therefore impossible to solve each system independently. The matrices are thus united within a global matrix. The system of global linear equations is solved by the computer (simple systems can be solved by hand and generally constitute learning exercises).

The PDE is solved at the nodes of the mesh, that is to say that the solution is computed at given points (discrete resolution) and not at each point of the domain $\Omega$. This requires being able to interpolate, that is to say to determine the values at any point from the values known at certain points. Polynomial functions are generally used.

A finite element is the data of an elementary cell and basic functions of the approximation space whose support is the element and defined in such a way as to be interpolative (see Basic Functions).

We see here three sources of error, that is to say of difference between the calculated solution and the real values:

The modeling of reality: The domain $\Omega$ generally corresponds to physical parts, the calculation is based on ideal (faultless) versions of the parts, the material and the boundary conditions; This source of error is not specific to the finite element method and can be taken into account by the constraint-resistance method.

The ideal and continuous geometry is replaced by a discrete geometry and the values are interpolated between points; the more spaced the points, the more the interpolation function may deviate from the reality, but conversely, a too fine mesh leads to extremely long computation times and requires computing resources (in particular random access memory). It is necessary to find a compromise between the cost of the calculation and the accuracy of the results.

In the case of numerical calculations, inevitable rounding errors occur, the numbers being represented by a finite number of bytes.

All the skill of the engineer is to master these errors especially:

By simplifying the geometry (defeaturing), by removing details that are far from the areas to be studied and having little influence on the result;

By choosing suitable meshes, for example, grids of the beam type for slender parts, or of the shell type for fine pieces, by cutting the piece so as to be able to make regular meshes on certain zones, by refining the mesh in the critical areas;

By having a critical look at the outcome.

Although there are many software applications that use this method to "solve" problems in various domains, it is important that the user has a good idea of what he or she is doing, including the choice of mesh size and type Of elements that must be adapted to the problem posed: No software does everything for the user and one must always keep a critical eye vis-à-vis approximate solutions.

For this purpose, error indicators and error estimators are available to adjust the various parameters. 


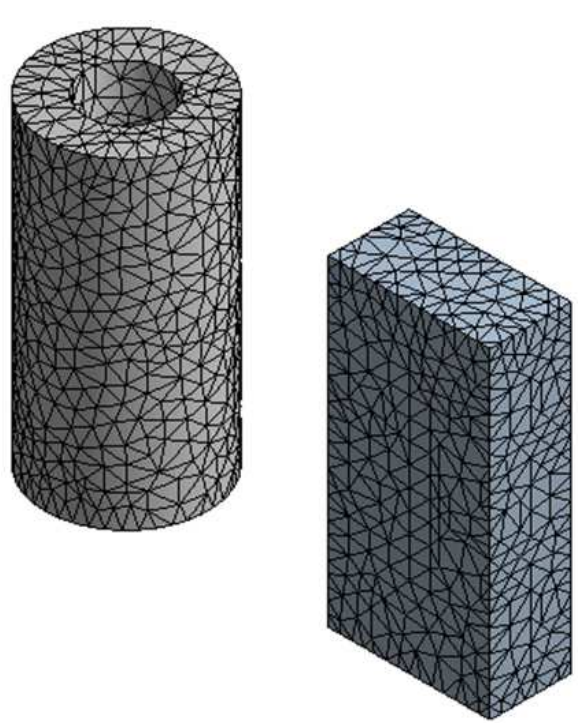

(a)

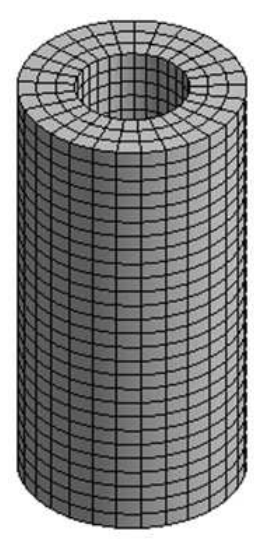

(b)

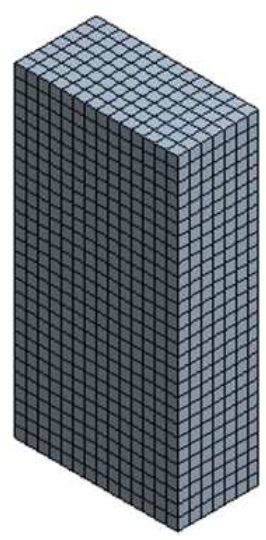

Fig. 7. The use of tetrahedral meshes (left) makes it possible to mesh "faithfully" complex geometries. a-the use of a regular mesh (right) is only possible on simple domains, b-but reduces the number of nodes and therefore the cost of the computation (Finite element method, from Wikipedia)

Once the solution has been found, it remains to determine the characteristics of the method thus developed, in particular the uniqueness of the possible solution or the numerical stability of the resolution scheme. It is essential to find a correct estimate of the error associated with the discretization and show that the method thus written converges, that is to say that the error tends to 0 if the fineness of the mesh also tends to 0 .

In the case of a linear PDE with a symmetric operator (as is the Laplacian operator), it is finally necessary to solve a linear algebraic equation, invertible in the best case.

The use of tetrahedral meshes (left) makes it possible to mesh "faithfully" complex geometries. The use of a regular mesh (right) is only possible on simple domains (a), but reduces the number of nodes and therefore the cost of the computation (b), (Fig. 7a and 7b).

\section{Conclusion}

Distributions of stress $3 \mathrm{D}$ in the vicinity of the overlapping corners of joints with a single lap were investigated with accuracy at present the man.

A (Fe), model accurately Finite Element 3D has been carried out in order to investigate the intensity of the stress of adjustments in the plan and outside of stress by the width of the plate.

The influences of the thickness of the adhesive and the Poisson distribution have been taken into account. Numerical results showed the presence of modes engaged has been detected with precision a sudden rise in the way the out-of-plan is very close to the free surface side of the fitting.

The adhesive joints were investigated in detail in the last decades and many investigations on this subject of research can be found easily in the past and the recent literature.

3D analysis FE a single tour-common with elastic properties of the material show the presence of modes coupled to the corners overlap. In particular, the sudden rise in the out-of-mode plan by the fracture in the vicinity of the free surface side of the joint is worthy of notice.

\section{Author's Contributions}

All the authors contributed equally to prepare, develop and carry out this manuscript.

\section{Ethics}

This article is original and contains unpublished material. The corresponding author confirms that all of the other authors have read and approved the manuscript and no ethical issues involved

\section{References}

Berto, F., P. Lazzarin, A. Kotousov and S. Harding, 2011. Out-of-plane singular stress fields in Vnotched plates and welded lap joints induced by inplane shear load conditions. Fatigue Fract. Eng. Mater. Struct., 34: 291-304.

DOI: $10.1111 /$ j.1460-2695.2010.01518.x 
Bogy, D.B., 1970. On the problem of edge-bonded elastic quarter-planes loaded at the boundary. Int. J. Solids Struct., 6: 1287-1313. DOI: 10.1016/0020-7683(70)90104-6

da Silva, L.F.M., P.J.C. das Neves, R.D. Adams and J.K. Spelt, 2009a. Analytical models of adhesively bonded joints-Part I: Literature survey. Int. J. Adhesion Adhesives, 29: 319-330. DOI: 10.1016/j.ijadhadh.2008.06.005

da Silva, L.F.M., P.J.C. das Neves, R.D. Adams and J.K. Spelt, 2009b. Analytical models of adhesively bonded joints-Part II: Comparative study. Int. J. Adhes. Adhesives, 29: 331-341. DOI: $10.1016 / \mathrm{j} . \mathrm{ijadhadh} .2008 .06 .007$

Finite element method, from Wikipedia, the free encyclopedia.

Gross, R. and A. Mendelson, 1972. Plane elastostatic analysis of V-notched plates. Int. J. Fract. Mechan., 8: 267-276. DOI: 10.1007/BF00186126
Lazzarin, P. and M. Zappalorto, 2012. A three-dimensional stress field solution for pointed and sharply radiused Vnotches in plates of finite thickness. Fatigue Fract. Eng. Mater. Struct., 35: 1105-1119. DOI: $10.1111 / \mathrm{j} .1460-2695.2012 .01698 . \mathrm{x}$

Lazzarin, P., M. Quaresimin and P. Ferro, 2002. A twoterm stress function approach to evaluate stress distributions in bonded joints of different geometries. J. Strain Anal. Eng. Des., 37: 385-398. DOI: $10.1243 / 030932402760203856$

Pook, L.P., 1992. Finite element analysis of corner point displacements and stress intensity factors for narrow notches in square sheets and plates. Int. J. Fatigue, 53: 3-8. DOI: 10.1046/j.1460-2695.2000.00358.x

Pook, L.P., 2000. Crack profiles and corner point singularities. Fatigue Fract. Eng. Mater. Struct., 23: 979-992. DOI: 10.1046/j.1460-2695.2000.00249.x

Williams, M.L., 1952. Stress singularities resulting from various boundary conditions. J. Applied Mechan., 19: 526-528. 